\section{La anticoncepción de emergencia en América Latina y el Caribe}

\author{
Antonieta Martin ${ }^{1}$
}

Palabras clave: anticoncepción postcoital, planificación familiar, derechos de la mujer.

\footnotetext{
Population Council/Fronteras de la Salud Reproductiva, Oficina Regional para América Latina y el Caribe, Panzacola 62 - Escondida 110, Villa Coyoacán, 04000 México, D.F., México; teléfono: 5255599998651; correo electrónico: tmartin@popcouncil.org.mx
}

La tarea de introducir en los países de América Latina los anticonceptivos que se usan después del coito, conocidos como anticonceptivos de emergencia, no ha sido fácil. Entre los elementos que han contribuido a limitar el acceso a la información y el uso de la anticoncepción de emergencia, se destacan la Iglesia católica y los grupos de derecha, cuya presencia se ha incrementado en años recientes en los países latinoamericanos. Estas entidades han puesto en juego todo su poder para impedir que el uso de esos anticonceptivos se incluya en las normas y políticas de salud nacionales. Por su parte, los grupos de mujeres y las organizaciones de la sociedad civil han sabido enfrentar esa lucha, que en algunos países ha sido larga y difícil. Una excepción es el Brasil, país representativo de una sociedad abierta, donde no hubo oposición a la introducción de los anticonceptivos de emergencia.

\section{PROBLEMAS COMUNES RELACIONADOS CON LOS ANTICONCEPTIVOS DE EMERGENCIA}

En los países de la Región de las Américas, la introducción del método de anticoncepción después del coito en los servicios de planificación familiar, tanto públicos como privados, se ha visto obstaculizada por una serie de problemas comunes. Por un lado, hay trabas y obstáculos para conseguir que el método se mencione y se mantenga en las normas oficiales de los servicios de planificación familiar y, por otro, que las pastillas se provean en programas públicos y privados y en las farmacias.

La mayor dificultad proviene de los grupos de extrema derecha y otros afiliados a la Iglesia católica en la esfera de influencia política, los cuales se oponen a los anticonceptivos de emergencia por considerarlos abortivos. Lamentablemente, la misma percepción errónea existe entre muchos proveedores de servicios de salud al igual que en algunos sectores de la población. Es evidente que hace falta capacitar apropiadamente a los proveedores de servicios de salud y difundir información correcta a las posibles usuarias acerca de los mecanismos de acción y beneficios de ese método anticonceptivo.

Debido a las objeciones de grupos mal informados, hay problemas para registrar y distribuir los productos dedicados. En consecuencia, en un número considerable de países, esos productos no se consiguen en las farmacias, su venta está restrin- 
gida y el precio es muy alto. Como tampoco están disponibles en los servicios de salud públicos, el acceso a ellos está vedado a las poblaciones vulnerables y de escasos recursos.

En general, en América Latina no existe suficiente información sobre cómo y por qué se usa el método poscoital. Con objeto de introducir los anticonceptivos de emergencia, en muchos estudios iniciales se revisó el grado de conocimiento de los proveedores de servicios de salud. Sin embargo, no hay publicaciones sobre la situación actual, especialmente en los países que apenas comienzan a introducir el método. Aún falta investigar diversos aspectos del tema. No se han publicado estudios recientes que aborden cómo esos anticonceptivos afectan al uso del condón o de otros métodos anticonceptivos regulares. Tampoco se conocen investigaciones sobre cuán accesible es ese método para las adolescentes, jóvenes y adultas de escasos recursos y las que viven en zonas rurales. Por otro lado, es muy importante saber cuán disponibles están dichos anticonceptivos en los servicios de salud públicos. Estos problemas son de gravedad variable y se han enfrentado de diversas maneras. Los resultados han sido distintos en cada país de la Región.

\section{CONFERENCIA REGIONAL SOBRE EL DERECHO A LA ANTICONCEPCIÓN DE EMERGENCIA EN AMÉRICA LATINA}

La creación del Consorcio Latinoamericano de Anticoncepción de Emergencia (CLAE) en octubre de 2000 marcó un punto de avance importante para los grupos que defienden los derechos sexuales y reproductivos de las mujeres. Dos años más tarde, el CLAE convocó a una conferencia regional que se realizó en Quito, Ecuador, con el título "Derecho a la Anticoncepción de Emergencia en América Latina". Los principales objetivos de la conferencia fueron 1) incrementar el conocimiento y la aceptación de ese tipo de anticoncepción como un método seguro y eficaz para prevenir embarazos no deseados y 2) apelar a los encargados de las políticas de interés público para que apoyaran la inclusión de la anticoncepción de emergencia en las normas oficiales de planificación familiar de los países de la Región.

A la conferencia asistieron más de 120 participantes de 20 países, entre los que había parlamentarios, académicos y representantes de organismos gubernamentales y no gubernamentales, de grupos de mujeres y de jóvenes, y de los medios de comunicación. La conferencia tuvo un impacto importante en la coordinación de los esfuerzos y actividades de organizaciones que trabajan en pro de la salud reproductiva y los derechos sexuales y reproductivos de la mujer. Antes del encuentro, solo 10 países habían incorporado la anticoncepción de emergencia en sus normas de planificación familiar y en 13 había productos dedicados. A principios del 2004, 18 países habían registrado los anticonceptivos como norma oficial y 19 habían registrado productos dedicados. En ciertos países se constituyeron consorcios nacionales para lograr una mayor articulación entre las instituciones e influir en las políticas de salud y el acceso a la anticoncepción postcoital. Así se hizo en Argentina, Bolivia, Ecuador, Chile, Guatemala y Nicaragua. En otros casos, se formaron entidades como la Rede Brasileira de Promoção de Informação e Disponibilização da Contracepção de Emergência (Rede CE) en el Brasil y la Mesa de Vigilancia Ciudadana en Derechos Sexuales y Reproductivos en el Perú.

A partir de la conferencia, el apoyo de fundaciones y gobiernos donantes ha permitido incorporar a adolescentes y jóvenes de ambos sexos para que, en el marco de los derechos sexuales y reproductivos, tengan acceso al método y a la participación en la toma de decisiones y los procesos de elaboración de políticas públicas que les conciernen. Con ese objeto, el CLAE convocó a tres encuentros juveniles en tres subregiones de América Latina: 1) Centroamérica, México y el Caribe de habla hispana; 2) los países andinos, y 3) los países del Cono Sur. Después de asistir a los encuentros, grupos de jóvenes han seguido coordinando esfuerzos para potenciar sus peticiones, participar en foros nacionales e internacionales y organizarse en redes que hacen un seguimiento de los compromisos e intercambian información y experiencias.

\section{ESTUDIOS PARA INTRODUCIR LA ANTICONCEPCIÓN DE EMERGENCIA EN ALGUNOS PAÍSES}

Cuando se formó el Consorcio Internacional de Anticoncepción de Emergencia (ICEC por su sigla en inglés) en los Estados Unidos de América, se iniciaron algunos estudios con el fin de identificar las estrategias más adecuadas para introducir ese método en algunos países (1). Una de las primeras encuestas sobre conocimientos, actitudes y prácticas al respecto se llevó a cabo en México. Como resultado, las estrategias propuestas para introducir el método fueron: el cabildeo entre políticos y tomadores de decisiones; la capacitación de prestadores de servicios de salud; una línea telefónica para consultas sobre los anticonceptivos de emergencia, y un portal en la internet con información detallada sobre los fármacos. También se realizaron campañas de promoción en los medios de comunicación, como la radiodifusión de anuncios cortos, y se distribuyeron carteles, tarjetas y otros materiales. 
Para medir el impacto de todas esas actividades, se hicieron dos encuestas: una de base en 1997 y una de seguimiento en 2000. Los resultados mostraron muy poco conocimiento del método entre las mujeres. Por ejemplo, durante la primera encuesta, solo $13 \%$ de ellas habían oído mencionar los anticonceptivos poscoitales, mientras que en la encuesta de seguimiento $32 \%$ sabían de qué se trataban. De los proveedores de servicios de salud encuestados en 1997, solo $88 \%$ sabían algo del método, pero en 2000 todos estaban enterados. Además, en la encuesta de seguimiento, $93 \%$ de los proveedores opinaron que era factible ofrecer los anticonceptivos en México, frente a $31 \%$ de la encuesta inicial $(1,2)$.

Otro estudio pionero se realizó en el Brasil, Chile y México, para conocer cuánta aceptación tenían los anticonceptivos de emergencia. Los resultados de las entrevistas mostraron que, mientras más se conocía el método, más positiva era la opinión que se formaba la mayoría debido a la reducción potencial de embarazos en adolescentes, embarazos no deseados y abortos en condiciones de riesgo (3).

El embarazo en adolescentes es un grave problema no solo en la vida de la mujer y el hombre involucrados, sino también en la perspectiva de la salud pública. Sin embargo, es un tema muy sensible, difícil de discutir abiertamente. En el seno de las familias latinoamericanas, las discusiones entre padres e hijos sobre la sexualidad suelen ser escasas o nulas. Es bastante común que los padres no estén preparados para responder a las inquietudes de sus hijos. Muchos países aún se niegan a incluir la educación sexual en las escuelas y, cuando se incluye, la mayoría de los maestros carecen de la capacitación necesaria para discutir los temas o responder a las preguntas de los niños y adolescentes (4).

La realidad es que las adolescentes están expuestas a tener relaciones sexuales ocasionales, durante las cuales les es difícil hablar de anticoncepción o negociar el uso del condón. Si tuvieran acceso a los anticonceptivos de emergencia, tendrían mayor control para decidir si desean embarazarse o no.

La idea de que los anticonceptivos de emergencia provocan abortos ha tenido gran peso en América Latina, especialmente porque muchas sociedades son conservadoras y la Iglesia católica es muy poderosa. La única excepción es el Brasil, que cuenta con una sociedad más liberal y en donde se aceptó públicamente que el método postcoital previene la ovulación, inseminación o implantación, pero no provoca el aborto (5).

Un estudio realizado en el año 2000 con 43 organizaciones afiliadas a la Federación Internacional de Planificación de la Familia (IPPF, en inglés) muestra que en 23 países de América Latina y el Caribe esas organizaciones ya estaban ofreciendo servicios de anticoncepción de emergencia con el régi- men de Yuzpe, ya que los productos dedicados solo estaban disponibles en cuatro países (Argentina, Brasil, Jamaica y Venezuela). El régimen de Yuzpe lleva el nombre del médico canadiense A. Albert Yuzpe, que en 1974 publicó los primeros estudios sobre las píldoras de anticoncepción de emergencia, una combinación de estrógenos y progestágenos, que se administra en dos dosis, una dentro de las primeras 72 horas después de la relación sexual sin protección y otra 12 horas más tarde. La mayoría de las organizaciones afiliadas a la IPPF no experimentaron restricciones para ofrecer el método y no hubo actos hostiles de parte de sus gobiernos, pero sí de los grupos defensores de la vida (6).

\section{ACTIVIDADES Y SITUACIÓN EN DIFERENTES PAIISES}

\section{Argentina}

Después de la conferencia internacional en Quito, se integró el Consorcio Argentino de Anticoncepción de Emergencia (CAAE). El CAAE se ocupa de los derechos sexuales y reproductivos; los mecanismos de acción de los anticonceptivos y la inclusión de estos en las políticas de salud; la educación sexual y la prevención de la violencia en las escuelas; los adolescentes como transmisores de información educativa, y la anticoncepción de emergencia para ayudar a las mujeres que han sido víctimas de violencia sexual. Sin embargo, Argentina también tuvo que enfrentar los embates de la derecha. El año anterior a la formación del CAAE, la Corte Suprema de Justicia había resuelto retirar de la venta los anticonceptivos de marca Inmediat, por considerarlos abortivos. El fallo en contra de Inmediat sienta precedente, ya que nunca se habían prohibido por vía judicial otros métodos anticonceptivos previamente autorizados por el Ministerio de Salud (7).

A principios de 2003, se aprobó una ley que permite la distribución libre de anticonceptivos en Argentina. Pero una juez de la provincia de Córdoba prohibió el uso de los anticonceptivos orales y el dispositivo intrauterino argumentando que violan los derechos humanos de las mujeres. En las distintas provincias argentinas, las ONG siguen recibiendo ataques de los sectores religiosos, legislativos, académicos y profesionales que se oponen a los derechos sexuales y reproductivos y al uso de anticonceptivos de emergencia.

\section{Bolivia}

A partir de la conferencia de Quito, en Bolivia se creó un consorcio sobre la anticoncepción post- 
coito y el Ministerio de Salud integró los anticonceptivos a las prestaciones del seguro universal maternoinfantil (SUMI), lo que implica que deben entregarse gratuitamente a las personas que los soliciten. Asimismo, se aprobó la comercialización de un producto dedicado, Inmediat N. Desde noviembre de 2003, Casa de la Mujer y Marie Stopes Bolivia recogen información estadística sobre el uso del método en sus dos centros de salud en Santa Cruz, el de Cochabamba y el de Sucre.

Desde noviembre de 2003 hasta junio de 2004 se han distribuido 361 dosis de los anticonceptivos de emergencia. El perfil de la mayoría (65\%) de las usuarias es el de una mujer joven de 15 a 24 años o estudiante universitaria que tiene relaciones sexuales esporádicas y que las ha tenido sin protección. Entre las que acudieron a solicitar los anticonceptivos, $5 \%$ mencionaron la violación como razón.

Los varones que acudieron representaron $24 \%$ de los solicitantes y $10 \%$ de estos fueron con su pareja. Estos datos indican que los hombres jóvenes están participando junto con su pareja en las decisiones de salud reproductiva.

La mayor parte $(72 \%)$ de las usuarias eran universitarias y $24 \%$ eran estudiantes de secundaria o escuelas técnicas. Se habían informado acerca de la anticoncepción de emergencia en los medios de comunicación (22\%) y en los servicios de salud $(38 \%)$, así como en folletos o actividades del ICEC $(12 \%)$. Las mujeres que solicitaron el servicio por primera vez conformaban $77 \%$ del total; las que fueron por segunda vez, $20 \%$; y las que volvieron por tercera o cuarta vez, $3 \%$. A pesar de que cuando solicitan por primera vez se les asesora sobre el uso del condón y otros métodos, las mujeres no los aceptan porque no tienen parejas estables y el condón es objeto de prejuicios sociales. (Torre Cañal R, Marie Stopes Bolivia; comunicación personal.)

\section{Chile}

El Ministerio de Salud de Chile ha propugnado la norma de "paternidad responsable" desde 1993. Incluye la anticoncepción postcoital en dosis distintas de las que se especifican para el régimen de Yuzpe pero, además de no estar actualizado, el método tampoco está realmente al alcance. Es por esto que el Ministerio de Salud, el Instituto Chileno de Medicina Reproductiva (Icmer) y la Asociación Chilena de Protección de la Familia (Aprofa) están elaborando una nueva norma de regulación de la fertilidad, en la que se incluyen indicaciones correctas para el uso de la anticoncepción de emergencia.

Por otra parte, el lanzamiento de las Normas y guía clínica para la atención en servicios de urgencia de personas víctimas de violencia sexual, resultado de un trabajo conjunto del Ministerio de Salud y organizaciones de la sociedad civil, significó en la práctica la distribución gratuita del producto dedicado Postinor2 a las mujeres víctimas de violación que lo soliciten en los servicios de urgencia públicos. Esto ha desatado un fuerte debate con la Iglesia católica y con autoridades ligadas a grupos conservadores, que se oponen terminantemente al uso del método, ya que consideran que "es un abortivo que atenta contra el que está por nacer", sin tomar en cuenta el derecho humano básico de las personas de decidir acerca de su cuerpo y de su vida sexual y reproductiva.

Por su parte, el Icmer ha continuado la línea de investigación sobre los mecanismos de acción de los anticonceptivos de emergencia. Sus estudios, realizados en monas y ratas, indican claramente que el levonorgestrel no altera los procesos que ocurren después de la fecundación (8). Esta es la hormona que contienen las píldoras de anticoncepción de emergencia más modernas, cuya eficacia supera la del régimen combinado de Yuzpe. Las píldoras que contienen solo levonorgestrel se pueden tomar en dos dosis de $0,75 \mathrm{mg}$ cada una: la primera dentro de las 72 horas después del coito sin protección y la segunda, 12 horas después. Estudios recientes indican que las píldoras de levonorgestrel se pueden tomar en una dosis única de 1,5 mg y que su acción protege contra el embarazo hasta cinco días después de la relación sexual sin protección, si bien la eficacia de los anticonceptivos de emergencia disminuye a medida que pasan los días. El Icmer ha difundido esos resultados en diversos foros científicos y al público en general a través de los medios de comunicación.

Lamentablemente, los dos productos dedicados que se han registrado en Chile, Postinal y Postinor-2, han sido blanco de acciones judiciales por parte de los mismos grupos conservadores ligados a la Iglesia católica. La venta de Postinal fue prohibida por la Corte Suprema, y Postinor-2 está actualmente en litigio. Varias organizaciones de mujeres y otras que trabajan en el campo de la salud y los derechos reproductivos han tratado de hacerse parte (Postinal) o se han hecho parte (Postinor-2) en los procesos judiciales. De momento, Postinor-2 se puede vender en farmacias y distribuir gratuitamente en los servicios de urgencia, pero la situación futura es incierta. (Schiappacasse V, Icmer; comunicación por correo electrónico.)

\section{Colombia}

En 1995, la Asociación Pro-Bienestar de la Familia Colombiana (Profamilia), institución pionera en los servicios de planificación familiar y salud reproductiva en la Región, ya estaba capacitando al personal clínico en Bogotá para ofrecer servicios de 
anticoncepción de emergencia usando el régimen de Yuzpe; dos años después extendió la capacitación al resto del país. En 1998, se iniciaron las negociaciones para distribuir Postinor-2 en Colombia. En el año 2000, se incluyó la anticoncepción de emergencia en las normas de salud y se aceptó el registro oficial del producto dedicado Postinor-2, el cual es distribuido por Profamilia en sus clínicas y farmacias. Actualmente, Profamilia tiene 35 clínicas que atienden a 50\% de los usuarios de servicios de planificación familiar en el país.

Por otra parte, después de que el Instituto Nacional de Vigilancia de Medicamentos y Alimentos (Invima), órgano regulador de los medicamentos en Colombia, aprobó el registro de Postinor-2, Profamilia inició su campaña de promoción del método por radio y elaboró materiales educativos para las usuarias. Sin embargo la Iglesia católica, que siempre se ha opuesto a cualquier método anticonceptivo moderno, también ejerció presión y declaró que el anticonceptivo de emergencia es abortivo, lo que forzó al Invima a revisar el proceso de registro. Profamilia no dejó de distribuir Postinor-2, pero durante ese período hubo siempre la preocupación de que se pudieran terminar los insumos, o que se llegara a prohibir el método. Año y medio después, el Invima confirma que Postinor-2 es un método anticonceptivo optativo y mantiene su registro. Al igual que en el resto de la Región, el reto de Profamilia consiste en continuar informando acerca del método y hacerlo accesible a las poblaciones más vulnerables, como son las adolescentes, las jóvenes y otras posibles usuarias las zonas rurales más apartadas (1).

\section{Ecuador}

En Ecuador, durante 1997 cinco ONG empezaron a ofrecer los anticonceptivos de emergencia en sus servicios de salud. Se hicieron también las primeras investigaciones nacionales sobre los conocimientos y prácticas del método. En 1998, un grupo de ONG apoyó la revisión de las normas de salud reproductiva y logró que se incorporaran a estas los anticonceptivos. A partir de ese suceso, los proveedores de servicios de salud contaron con el respaldo legal y programático para informar acerca de, prescribir o administrar el método. Hasta la fecha, solo se cuenta con el método de Yuzpe, pero se espera muy pronto utilizar un producto dedicado.

En el Centro Médico de Orientación y Planificación Familiar (Cemoplaf), las usuarias más frecuentes de los anticonceptivos de emergencia son adolescentes solteras de clase media. Los registros muestran que en cada uno de los 28 centros de Cemoplaf se entregan entre 25 y 30 dosis al mes y no se ha visto ninguna relación negativa con el uso del condón u otro anticonceptivo. Las mujeres que los solicitan reciben muy buena orientación, con énfasis en la importancia de usar un método regular.

Otras actividades en Ecuador están dedicadas a apoyar a las mujeres que viven situaciones de violencia y a los jóvenes de ambos sexos en general. Se ha creado el Consorcio Ecuatoriano de Anticoncepción de Emergencia y todas las agencias que lo integran trabajan en coordinación con el Ministerio de Salud Pública, el Ministerio Fiscal y la Policía Nacional.

\section{Honduras}

Desde 1999, en Honduras se incluye la anticoncepción de emergencia en las normas de planificación familiar, pero hasta agosto de 2004 no existía un producto dedicado registrado. La Asociación Hondureña de Planificación de la Familia (Ashonplafa) y otras instituciones hondureñas asistieron a la conferencia en Quito, en donde elaboraron planes de trabajo para mejorar la agenda de los derechos sexuales y reproductivos en Honduras. Con financiamiento de la Fundación Summit, apoyo técnico del Population Council, y la colaboración de Marie Stopes Internacional (MSI) y Ashonplafa, se realizaron encuestas de conocimientos, actitudes y prácticas de jóvenes de 15 a 20 años de edad en los colegios y escuelas secundarias de La Ceiba y Tegucigalpa, así como de usuarias de clínicas de planificación familiar en San Pedro Sula y Tegucigalpa, y estudiantes de la Universidad Nacional Autónoma de Honduras.

En el marco de ese proyecto, se elaboraron 10000 paquetes especiales de anticonceptivos de emergencia que contenían el régimen de Yuzpe, más condones y material informativo para su uso. Un año después de su introducción, se habían distribuido alrededor de 1500 paquetes. El paquete cuesta 30 lempiras, alrededor de US\$1,57, y está en venta en clínicas de Ashonplafa, MSI y puestos de salud de las maquiladoras (9). Además, Ashonplafa y MSI han capacitado a proveedores de servicios, vendedores de farmacias y periodistas y han producido materiales educativos y audiovisuales, así como cuñas radiales con mensajes dirigidos a adolescentes, mujeres adultas y trabajadoras de maquila.

A principios de 2004, se desató una controversia con los grupos conservadores y de la Iglesia católica, provocada por la discusión en medios de comunicación de la inclusión de la anticoncepción de emergencia en las normas de salud de México. Ashonplafa y MSI continuaron informando y distribuyendo el método y recibieron apoyo del Fondo de Población de las Naciones Unidas (FNUAP) y de los grupos de mujeres para ampliar la difusión y el 
acceso a los anticonceptivos. Por lo pronto, la anticoncepción de emergencia sigue incluida en las normas y distribuyéndose según el régimen de Yuzpe en las clínicas de Ashonplafa y MSI.

\section{México}

A partir de 1974, el gobierno de México adoptó políticas progresistas de planificación familiar. La tasa global de fecundidad bajó de 6,7 hijos por mujer en el decenio de 1970 a 2,5 en 1995. Por eso llama la atención que la anticoncepción de emergencia haya pasado por un proceso tan largo de aprobación, que duró más de 10 años. No obstante la alta prevalencia del uso de métodos anticonceptivos (mujeres urbanas, $72 \%$; rurales, $52 \%$ ), sigue registrándose un porcentaje alto de embarazos no deseados, especialmente entre las adolescentes que empiezan a tener relaciones sexuales desprotegidas.

El proyecto de norma de los servicios de planificación familiar, que incluía la anticoncepción de emergencia como una opción, se elaboró en 1993, pero permaneció inactivo por muchos años. A fines de 2003, las autoridades de salud solicitaron la aprobación de la norma, la cual se obtuvo en enero de 2004. Esto desató un gran debate con los grupos conservadores y la Iglesia católica. Las autoridades de salud, las sociedades médicas y científicas, los grupos de mujeres, los medios de comunicación y la sociedad en general apoyaron la idea de tener una opción anticonceptiva postcoital.

Actualmente hay cuatro productos dedicados disponibles en la mayoría de las farmacias, a precios poco accesibles para las mujeres de escasos recursos (entre US\$ 5 y 20). Por su parte, los servicios públicos no los tienen, aunque algunas ONG los ofrecen en sus clínicas y farmacias a precios subsidiados que oscilan entre $\$ 5$ y $\$ 6$.

En los sondeos de opinión pública realizados por los medios de comunicación en los días posteriores a la entrada en vigor de la norma, la población se opuso 3 a 1 a las opiniones de los líderes conservadores y de la Iglesia católica. Un comentarista del programa de noticias radiofónicas matutinas preguntó: “¿Qué va a hacer la Iglesia católica del mundo para enfrentar los nuevos tiempos, para enfrentar la realidad de una nueva manera de pensar de las generaciones que están viniendo?" (Carlos Loret de Mola, W Radio).

Los grupos conservadores todavía interpusieron una demanda judicial, pidiendo el "amparo" contra la norma. El juez determinó que la norma oficial mexicana 005-SSA2-1993 que regula los servicios de planificación familiar "por su sola entrada en vigor, no causa ningún perjuicio, ya que no im- pone a los ciudadanos ninguna obligación de acatarla." El juez señaló que "de ningún modo puede considerarse agravio o afectación a la persona o a los derechos de quienes promovieron el amparo la entrada en vigor de esa norma" (10).

\section{Paraguay}

En Paraguay, la anticoncepción de emergencia se incluyó en las normas del Ministerio de Salud Pública y Bienestar Social en enero de 1998, cuando se ofrecía con el régimen de Yuzpe. Antes de la conferencia de Quito de 2002, ya había productos dedicados registrados. En el año 2001, el FNUAP había donado 40000 dosis de Postinor-2 para distribución gratuita en los servicios de salud pública.

Pero Paraguay tampoco fue la excepción en la oposición de grupos de poder conservadores. En 2002, durante el Congreso Latinoamericano de Salud Sexual y Reproductiva convocado en Buenos Aires por la Asociación Argentina por la Salud Sexual y Reproductiva (Asser), cuando Promoción y Mejoramiento de la Salud (Promesa) anunció su proyecto de mercadeo social de los anticonceptivos, hubo fuerte oposición y solicitudes para que se interrumpiera el proyecto. Sin embargo, el propio Ministerio de Salud apoyó a Promesa para que continuara con su proyecto. Por otra parte, la Cámara de Farmacias del Paraguay (Cafapar) y Promesa realizaban, y siguen haciéndolo, talleres de actualización en tecnología anticonceptiva para proveedores de servicios de salud, con énfasis especial en los anticonceptivos de emergencia.

A principios de 2003, Promesa registró un anticonceptivo de emergencia, producto de mercadeo social, que se llama Pronta. A partir de febrero de 2004 , Pronta se hizo accesible en $75 \%$ de las farmacias del país. Hasta junio de 2004 se habían distribuido 3600 dosis. Las otras marcas comerciales, Inmediat $\mathrm{N}$ y Control, venden un promedio de 1000 dosis al mes a pesar de que el precio es elevado, entre $\$ 4,40$ y $\$ 5,05$, respectivamente. Datos de Promesa indican que después de las actividades de capacitación para proveedores de servicios de salud y de información y educación para los jóvenes, el Ministerio de Salud incrementó la distribución de Postinor-2 de 217 dosis en el año 2001 a 1749 dosis en 2003 (11).

\section{Perú}

Perú, al igual que otros países de la Región, tiene tasas altas de embarazos no deseados, abortos arriesgados y mortalidad materna. Al mismo tiempo, la discriminación social y cultural contra las mujeres les niega acceso a los servicios de salud re- 
productiva. El grupo de edad de las mujeres adolescentes es el más vulnerable, ya que enfrentan más barreras para acceder a los servicios; por eso, la disponibilidad de anticonceptivos de emergencia se convierte en un tema cada vez más crítico en este y otros países de la Región (12).

Después de la Conferencia Internacional sobre Población y Desarrollo que tuvo lugar en El Cairo en 1994 y la Cuarta Conferencia sobre la Mujer en Beijing en1995, el gobierno peruano, al igual que la mayoría de los países latinoamericanos, apoyó los servicios de salud reproductiva e incorporó la anticoncepción oral de emergencia en las normas nacionales de planificación familiar. No obstante, las píldoras nunca estuvieron disponibles en el mercado o en los servicios de salud. Por razones de índole política, a fines del decenio ese tipo de anticoncepción se suprimió de las normas nacionales (13).

En 1999, varias ONG se coordinaron con el Centro de la Mujer Peruana Flora Tristán con el fin de lanzar una campaña en los medios para promover el conocimiento y uso de los anticonceptivos de emergencia, especialmente entre la población joven de Lima. A principios de 2001, el Population Council y la Sociedad Peruana de Obstetricia y Ginecología (SPOG) organizaron un taller de capacitación sobre anticoncepción de emergencia para líderes de opinión y en julio de ese año el método postcoital se volvió a incorporar en las normas de planificación familiar. En 2001, la Dirección General de Salud aprobó la distribución y venta de Postinor-2.

Posteriormente, llegaron al Ministerio de Salud personas muy conservadoras que sostenían que los anticonceptivos de emergencia son abortivos y los retiraron de las normas. Para apoyar su decisión, solicitaron la opinión de la SPOG, órgano oficial del Colegio Médico, especializado en asuntos de salud sexual y reproductiva, que se pronunció a favor del uso del método postcoital. Mientras tanto, organismos de la sociedad civil iniciaron actividades de capacitación, promoción y mercadeo social para hacer accesible al público el producto dedicado Postinor-2. En los medios de comunicación aumentó la discusión sobre los derechos sexuales y reproductivos y se criticó a las autoridades de salud por imponer sus creencias religiosas en las políticas de salud.

Los parlamentarios también revisaron las políticas de salud reproductiva del Ministerio. Realizaron audiencias e invitaron a instituciones y líderes a presentar sus puntos de vista. Además de la SPOG, en esas actividades participaron el Instituto Peruano de Paternidad Responsable (Inppares), expertos nacionales e internacionales de Venezuela, Chile, México y Colombia, y grupos de mujeres.

En 2004, volvieron a incorporarse en las Guías de atención de salud sexual y reproductiva los anticon- ceptivos de emergencia y el Ministerio anunció que se distribuirían de manera gratuita en los centros de salud de todo el país. El Ministerio de Justicia dictaminó que el método no es abortivo y que no viola ninguna norma constitucional. Esto puso fin a un período de tres años de inequidad e injusticia, como comentaron algunas ONG.

\section{PASOS PARA EL FUTURO}

En la mayoría de los países de la Región se ha logrado avanzar en lo que se refiere al conocimiento del método anticonceptivo postcoital. Sin embargo, todavía se necesita trabajar en una serie de áreas importantes.

Es preciso continuar con la capacitación de proveedores de servicios de salud. Sobre todo, hay que seguir promoviendo los derechos sexuales y reproductivos de las mujeres y el acceso a los anticonceptivos de emergencia para prevenir embarazos no deseados y abortos riesgosos, principalmente entre las adolescentes que están en riesgo continuo de tener relaciones sexuales sin protección, así como en otros grupos vulnerables de mujeres de escasos recursos, que habitan en zonas rurales o pertenecen a grupos indígenas. No puede bajarse la guardia ante los grupos conservadores y de la Iglesia católica, que siguen siendo muy poderosos en los ámbitos económico y social y presionan a las autoridades de salud para que eliminen los anticonceptivos poscoitales de las normas de salud.

Es importante seguir trabajando con los medios de comunicación, ofrecer talleres para periodistas y preparar paquetes informativos para la prensa, radio y televisión. Por otro lado, debe continuar el trabajo de cabildeo en las comisiones de salud y de derechos humanos de los congresos para que respalden las iniciativas sobre derechos sexuales y reproductivos.

Se necesita buscar los mecanismos para que los anticonceptivos de emergencia se puedan obtener gratis en los servicios públicos de salud. También debe mejorarse la disponibilidad, el costo y la distribución de los productos dedicados en las farmacias y continuar haciendo estudios sobre las condiciones en que se usa el método poscoital y su relación con el uso del condón y otros métodos anticonceptivos.

\section{SYNOPSIS}

\section{Emergency contraception in Latin America and the Caribbean}

Introducing the post-coital birth control method in the familyplanning services of Latin American countries has not been 
an easy task. Catholic and other conservative groups with great influence in the political arena have time and again stopped it from being adopted as an alternative method and have even succeeded in having it removed from official directives after formal acceptance by health authorities. The main objections are triggered by the erroneous supposition that "emergency contraception" pills are abortifacients. However, a large dose of cultural discrimination against women seems also to be involved. It has been extremely difficult to register dedicated products and make them available in drugstores and even more difficult to distribute them without charge at public health centers. They are hard to find, expensive, and unavailable to adolescents at risk for unwanted pregnancies and to most low-income women, especially in rural areas. Dissemination of appropriate information has been scarce and slow and there are still great numbers of people that do not understand how or why the method works. Brazil has been the only exception, as its open society has readily accepted this method of contraception.
The Latin American Consortium on Emergency Contraception founded in the year 2000 and its regional conference two years later had an important impact on the situation, as they encouraged the coordination of efforts by governmental and nongovernmental entities with those of women's groups to fight for sexual and reproductive rights. A number of studies have shown that the more people learn about emergency contraception, the more they find it acceptable and necessary, and radio spots and other media techniques have begun to educate the public about this matter. In spite of the many difficulties encountered, in the last few years several countries have made strides to include this method in their public health guidelines. However, because of the powerful forces against it, accessibility and distribution of the emergency pills are not always implemented as planned and there are still many areas that require work. Details are given on the situation in Argentina, Bolivia, Chile, Colombia, Ecuador, Honduras, Mexico, Paraguay, and Peru.

\section{REFERENCIAS}

1. Heimburger A, Acevedo-García D, Schiavon R, Langer A, Mejía G, Corona G, et al. Emergency contraception in Mexico City: knowledge, attitudes and practices among providers and potential clients after a three-year introduction effort. Contraception. 2002;66:321-9.

2. Gerstein L. Providers, clients okay emergency contraception in Nairobi and Mexico City (digest). International Family Planning Perspectives. 2000;26(2):95-96.

3. Díaz S, Hardy E, Alvarado G, Ezcurra E. Acceptability of emergency contraception in Brazil, Chile, and Mexico, 1: Perceptions of emergency oral contraceptives. Cad Saude Publica. 2003;19(5):1507-17.

4. Martin A. Estudio de necesidades de información y servicios para adolescentes en el Instituto Mexicano del Seguro Social [informe inédito]. México, D.F.: Johns Hopkins University/Center for Communication Programs/Population Communication Services; 1999. (Proyecto financiado por USAID, 1993-2000.)
5. Díaz S, Hardy E, Alvarado G, Ezcurra E. Acceptability of emergency contraception in Brazil, Chile, and Mexico, 2 : Facilitating factors versus obstacles. Cad Saude Publica. 2003;(19):1729-37.

6. Díaz-Olavarrieta $C$, Norris Turner A, Ellertson C, Helzner JF, Ezcurra E. Policy climate, scholarship, and provision of emergency contraception at affiliates of the International Planned Parenthood Federation in Latin America and the Caribbean. Contraception. 2002;65:143-9.

7. Lubertino, MJ. Un fallo abortivo: la Suprema Corte de Justicia de la nación contra la anticoncepción de emergencia. Argentina: Instituto Social y Político de la Mujer; 2001 [comunicación recibida vía internet].

8. Ortiz ME, Ortiz RE, Fuentes MA, Parraguez $\mathrm{VH}$, Croxato HB. Post-coital administration of levonorgestrel does not interfere with post-fertilization events in the new-world monkey Cebus apella. Human Reproduction. 2004;19(6):1352-6.
9. García S, Lara D. Emergency contraception in Honduras: expanding knowledge, access and use [informe final inédito]. México, D.F.: Population Council; 2004.

10. Villamil J. Debate sobre anticonceptivo de emergencia podría convertirse en revés para la Iglesia. La Jornada, 28 de enero de 2004, Sección Política [diario nacional, México].

11. Kamm LF. Proyecto de marketing social de anticoncepción de emergencia (PAE) en Paraguay. ONG Promesa; Informe de actividades 2001-2004 [información parcialmente publicada en la revista INFOFARMA, 2004].

12. Coe A-B. Decisiones informadas: ampliando el acceso a la AE en Perú. Boletín del CLAE. 2002;1 (1):7-8.

13. Coe A-B, Chávez S. Informe sobre los avances de la anticoncepción oral de emergencia (AOE) en el Perú; 2003 [Informe inédito obtenido por comunicación electrónica]. 